\title{
Negative-pressure plastic isolator for patients with dangerous infections
}

\author{
P C TREXLER, R T D EMOND, BRANDON EVANS
}

British Medical fournal, 1977, 2, 559-561

\section{Summary}

A negative-pressure plastic isolator is effective for dealing with patients suffering from dangerous infections. So far it has been used to treat seven patients suspected of having infections due to Lassa, Marburg, or Ebola viruses. One patient spent 32 days in the isolator. The isolator has proved comfortable and acceptable to patients, and it gives the nursing and medical attendants a high degree of protection. All routine nursing and medical procedures can be carried out with minimal interference by the physical barrier, though it is not practicable to undertake artificial respiration or haemodialysis.

\section{Introduction}

An attempt has been made to reduce the hazard to attendants caring for patients with infections-for example, those caused by Lassa, Marburg, Ebola viruses-against which no immunisation is readily available. Laboratory experience since 1957 has shown the effectiveness of flexible-film isolators in maintaining microbial isolation of laboratory animals, and recently isolators have been used to protect neutropenic patients. ${ }^{1}$ The latter type

Royal Veterinary College, London NW1 0TU

P C TREXLER, MS, reader in veterinary gnotobiotics

Infectious Disease Department, Royal Free Hospital, Coppetts Wood Hospital, London N10 17N

$R$ T D EMOND, MB, FRCP, consultant in infectious diseases

BRANDON EVANS, $M B$, MRCP, senior registrar of isolator has been modified to protect attendants without interfering with the treatment and care of patients.

\section{Apparatus}

The isolator system ${ }^{2}$ (see figure) is the same size and shape as the reverse-barrier isolators used to protect infection-prone patients. A workspace and a bed with a walkway are placed within two separate but interconnected isolators. These isolators consist of envelopes, made of polyvinylchloride film, totally enclosing the isolated space. The supporting frames and trolley, the bed, mattress, table, and chair are on the outside of the envelope, but can be used in the usual way through the film. Manipulations within the isolated space are accomplished through attached gloves or half-suits forming invaginations of the isolating envelopes.

Air filters attached to the bed isolator prevent contamination of the ventilation system. An air-pressure regulator is adjusted to provide negative pressure within the envelope, preventing egress of airborne particles if the isolator is accidentally punctured. Sufficient negative pressure capacity to compensate for the air displaced by movement of the half-suits is provided by the sidewalls, which are suspended from the overhead frame and are free to move with the internal envelope pressure.

\section{SUPPLYING THE ISOLATOR}

All materials are introduced and removed through a single port on the side of the supply isolator. A tightly fitting polyethylene plastic bag is placed over the port from the outside and is held in place by a rubber band. To introduce materials the lip of the bag is slid to the edge of the port and another bag, containing the material to be introduced, is placed over the old bag and secured to the port by a rubber band. The old bag is pulled into the isolator and the materials introduced, leaving the new bag closing the port. Materials to be removed are placed in the bottom of the bag sealing the port and the neck of the bag tied-off by means of an externally applied strap. The internal surface of the bag in this area is swabbed with an appropriate germicide and a second strap applied. The bag is cut between the two straps 


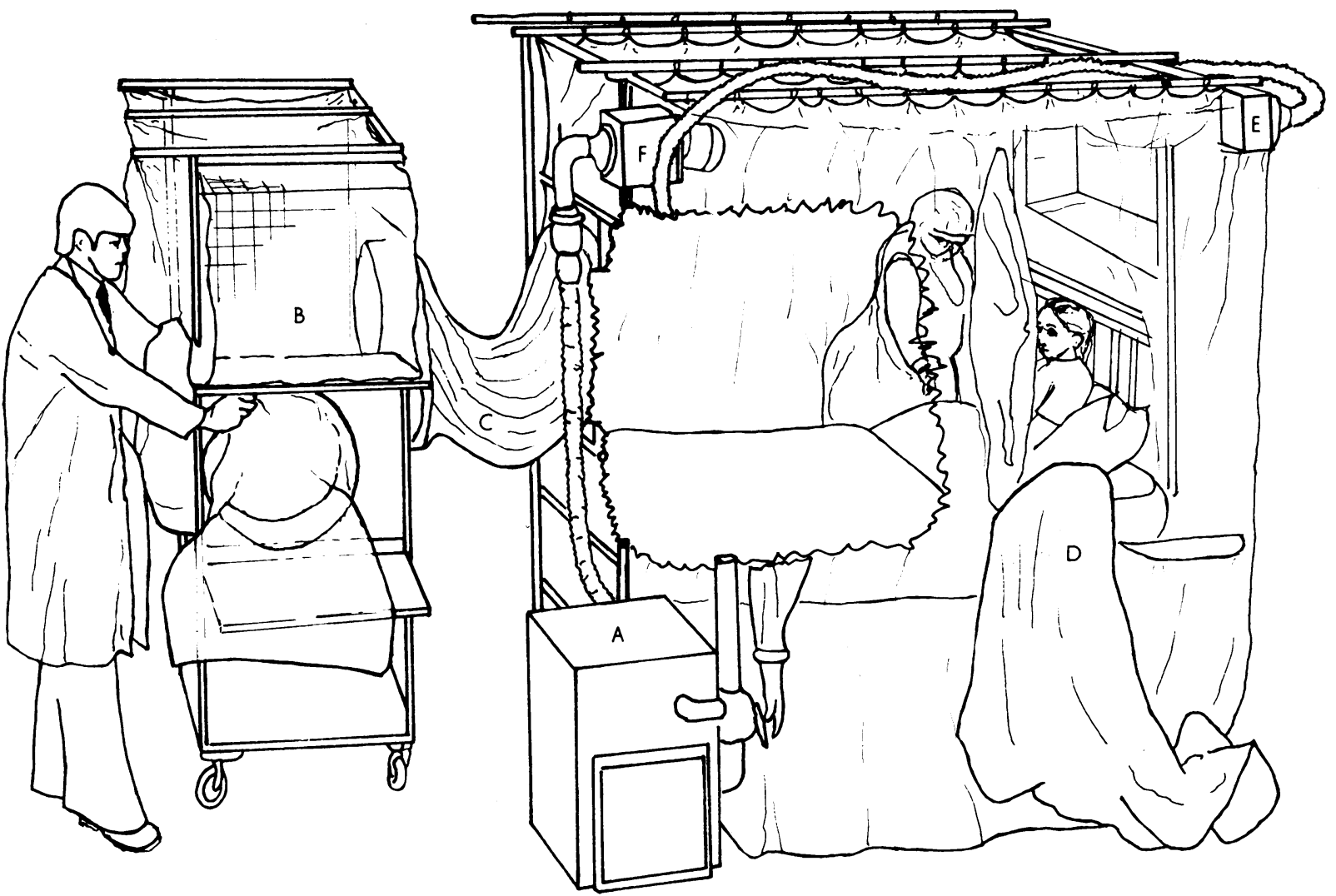

Containment bed isolator showing a patient in bed, a nurse in a half-suit, and the entry port on the supply trolley in use. $\mathrm{A}=\mathrm{Air}$ supply unit. $B=$ Supply trolley. $C=$ Attachment sleeve. $D=$ Half-suit. $E=$ Supply airfilter. $F=$ Exhaust airfilter.

using a hot wire cautery, leaving the port closed and the materials in a sealed bag. The sleeve connecting the two isolators can be managed in the same manner after the patient enters the bed isolator.

Wrist-length gloves are attached to the sleeves on the envelope walls and half-suits by means of rigid triple groove rings. Gloves are replaced from the outside by sliding the glove cuff to the distal groove and placing a new glove over the old and attaching the cuff to a proximal groove. The old glove is then pulled into the isolator and removed with other waste material. Wires and tubes can be passed through the envelope wall without risk of contamination by placing the end of the wire or tube, from the outside, into a plastic film cone located where needed. The tip of the cone is cut off from the inside of the isolator and the wire or tube pulled through and taped securely to seal the tip of the cone.

\section{Supportive services}

The isolator should be sited within a large room in a high-security unit to ensure safety should there be a major failure of the equipment or faulty technique in disposing of contaminated waste. Moreover, there is always the remote possibility that the patient may have to be removed from the isolator in the event of a major emergency, such as fire. There should be direct access for admission of the patient to the isolator and suitable changing and shower rooms for the staff.

The patient's room should be fitted with storage racks for supplies, which can be replenished daily as necessary. The high-security unit should be fully equipped for disposing of infected material by incineration or sterilisation and have facilities for entertainment during convalescence.

\section{Control of infection}

Before the patient is admitted the isolator is stocked with sufficient supplies to last for 24 hours. Thereafter fresh supplies are introduced as required, and all waste material is removed in sealed bags for incineration or sterilisation. Dry waste is removed as already described and immediately destroyed by incineration within the high-security area. Liquid waste, including washing water, mouth washes, surplus drinks, vomitus, urine, and fluid faeces, is emptied through a funnel into a 2.5-litre plastic bottle containing Hycolin, a creosolic disinfectant. The volume of disinfectant is adjusted to give a final concentration of $1 \%$. Full bottles are removed one at a time from the supply isolator in the usual manner then placed in an additional heatsealed bag to prevent accidental leakage. The liquid waste is stored for a minimum of 12 hours to allow time for the disinfectant to act and afterwards boiled before disposal. Medical staff disposing of liquid waste wear full protective clothing, including a visor and face mask.

If it is decided that a patient is not suffering from a dangerous infection the contents of the isolator are dealt with according to routine barrier-nursing procedures. The envelopes are subsequently sprayed with $1 \%$ hypochlorite solution and washed thoroughly before reuse. If it has been confirmed that the patient has had a dangerous infection all the contents of the isolator are removed for incineration or autoclaving. The interior of the isolator and the adjacent highsecurity area are fumigated with formaldehyde and left for 24 hours The envelopes are then detached from their frames, collapsed down under maximum negative pressure, and later burnt. The filters are autoclaved before incineration.

Should a patient die from a dangerous infection special precautions must be taken to prevent contamination of the environment. The ventilation system of the isolator should be kept running to maintain a negative pressure until the body has been removed. The sleeve connecting the supply isolator to the patient isolator is clamped in two places and divided. The supply isolator is then removed and a trolley brought up to the patient isolator. A large plastic bag is now placed over the stump of the sleeve and fixed in position over the rim of the entry port. The remains of the old sleeve are withdrawn into the isolator and the corpse lifted into the plastic bag, which is then clamped and separated, leaving a stump to seal the entry port. The body in the plastic bag is finally put in a coffin for cremation or burial.

\section{Medical care}

Separation by the flexible-film barrier does not seriously impair access and we have found it possible to carry out all routine medical

(1)

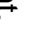


procedures, though we have had to modify certain aspects of clinical examination and treatment.

The plastic film tends to reduce the amount of light within the isolator, especially at night, and a supplementary fluorescent light fixed over the isolator greatly facilitates inspection of the patient. We have found an electronic stethoscope preferable to the standard aural instrument for it is more sensitive and effectively overcomes the background noise generated by the pump units. The wire from the chestpiece can be passed through one of the cones and sealed in position so that when required it can be plugged into a receiver with headphones outside the envelope. Other electronic monitoring equipment can be used in a similar manner, and it is our normal practice to install a cable for electrocardiograph leads. It is advisable to insert any wires and tubes into the isolator before use, bearing in mind that leads and instruments may have to be destroyed should the infection prove dangerous.

Auroscopy and ophthalmoscopy are possible but the instruments must be used within the envelope and difficulty may be experienced in focusing because the visor of the suit is interposed. Radiological examination is possible using a mobile set with the tube head inserted into a special sleeve incorporated in the side of the tent. The film cassette is inserted into a separate sleeve that is long enough to reach any part of the patient. Blood sampling and injections are carried out in the usual way but in view of the special risks needles should be placed in screw-topped hard plastic containers before removal.

Intravenous infusions present no problems. It is advisable to use plastic containers for the fluids so that they can be incinerated after use. All equipment for the infusion is used within the envelope and introduced as required. There are special sleeves welded into the roof of the envelope for the attachment of the infusion bags, and these can be adjusted to the required height. Peritoneal dialysis can be performed readily, but haemodialysis is not possible. Intubation and tracheostomy could be performed with some difficulty but artificial ventilation would necessitate a respirator within the isolator and is not practicable at present. Suction can be provided within the isolator by either a hand-operated sucker or an electric machine connected through a cone to an external power source. On no account should the sucker be sited outside the envelope.

\section{Experience}

Experience in the use of the isolator for containing infection was gained initially by nursing six volunteers who were suffering from various mild infectious diseases. These patients remained in the isolator for a combined period of 22 days. We encountered no serious difficulties and rectified several minor defects. Over the last year seven patients, suspected of having Lassa fever, Marburg disease, or Ebola virus disease, were nursed in the isolator over a period of 52 days. The patients were mainly young adults but included a child of 11 years. We have had no experience of nursing very young children or elderly patients in the isolator.

When patients were admitted for observation they were generally aware of their predicament and accepted the restrictions of the isolator as a necessary precaution. There were no problems with temperature or humidity provided that the isolator was not exposed to direct sunlight. Noise levels were acceptable and did not interfere with sleep or with listening to the radio or television. Communication between staff and patients was easy but conversation between staff wearing halfsuits and others outside the tent proved more difficult unless voices were raised. If prolonged, convalescence might be very trying because of the constraints of the space available for exercise. Much could be done to alleviate boredom by providing reading material, a radio and television set, and by maintaining a telephone link with the outside world. Although an occasional patient spent a prolonged period within the isolator, especially while waiting for the results of clearance specimens, most were removed within a few days once the diagnosis had been established. One of our patients spent 32 days in the isolator with no serious problems. ${ }^{3}$

Initially our nurses expressed some misgivings about looking after patients in the isolator but they gained confidence after experience had shown that the techniques were practicable and that patients could be effectively nursed within the isolator. Our staff now appreciate the protection afforded by the system and prefer to nurse potentially dangerous patients in this manner. At least 12 trained nurses are necessary to provide effective cover, and constant training is essential to ensure proficiency. This can be achieved by weekly training sessions and by partnering a newcomer with an experienced nurse when dealing with an actual patient. Basic skills are usually acquired within two days.

\section{References}

1 Trexler, P C, Spiers, A S D, and Gaya, H, British Medical fournal, 1975, 4, 549.

2 Trexler, P C, Postgraduate Medical fournal, 1976, 52, 545.

${ }^{3}$ Emond, R T D, et al, British Medical fournal, 1977, 2, 541.

(Accepted 29 June 1977)
The nipples of a young boy are placed abnormally high on his chest. Might this indicate chromosome abnormality?

It would be difficult to define abnormally high placement of a boy's nipples, but in any case it does not indicate a chromosome disorder.

\section{What is the treatment for caruncle?}

A caruncle is a granulomatous lesion appearing at the external urinary meatus, usually in the mid-posterior aspect. It is deep red in colour; a biopsy should be taken to confirm the granulomatous nature of the tissue, and it should respond satisfactorily to diathermy coagulation. Much more common than the caruncle is prolapse of the urethral mucosa in women, which is usually loosely referred to as a caruncle. The appearance is different in that the colour resembles that of the urethral mucosa and not the deep red colour of a true caruncle. Prolapse certainly can be treated by diathermy coagulation but will almost always recur after a period. In most patients a prolapse of the urethra causes minimal symptoms, but if it produces symptoms of discomfort in the perineum while sitting down or some disturbance of micturition, and temporary relief is achieved by local diathermy of the prolapse, then it can be cured more permanently by a small operation to extend the urethra so as to absorb the redundant mucosa. This operation is described as "urethral advancement."

$A$ patient aged 21 started taking levonorgestrel two months before her marriage. She has had no side effects from the pill, but has noticed a definite increase in the size of her breasts. Is this unusual?

Presumably levonorgestrel was prescribed as an oral contraceptive. It is a little surprising that this progestogen should have been given by itself alone. Usually D-norgestrel or DL-norgestrel is combined with ethinyloestradiol in oral contraceptive preparations. If an oestrogen was combined with the progestogen it is equally likely that the oestrogen was the agent responsible for the breast changes, as both types of steroid promote breast growth. Although patients on oral contraceptives often complain of breast changes, the usual effect is a temporary engorgement rather than permanent enlargement. Breast changes of various kinds are not unusual with patients on oral contraceptives and are certainly not limited to those containing norgestrel. Although galactorrhoea was not mentioned, a prolactin assay might be advisable. Many women with post-pill amenorrhoea have hyperprolactinaemiapossibly resulting from a hyperplasia of the prolactin-producing cells provoked by the oral contraceptive. 